\title{
El ganado bovino Criollo Coreño del occidente de México en la producción de carne: caracterización, retos y perspectivas
}

Guillermo Martínez-Velázquez ${ }^{\text {a }}$

Ángel Ríos-Utrera ${ }^{b}$

José Antonio Palacios-Fránquez ${ }^{\text {a }}$

Vicente Eliezer Vega-Murillo $^{c}$

Moisés Montaño-Bermúdez ${ }^{\mathrm{d}^{*}}$

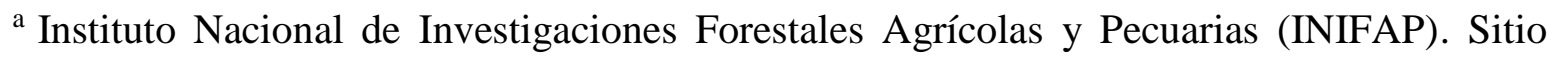
Experimental "El Verdineño", km 7.5 carretera Navarrete-Sauta, Municipio de Santiago Ixcuintla Nayarit, México.

${ }^{\mathrm{b}}$ INIFAP, Campo Experimental La Posta, Veracruz, México.

c Universidad Veracruzana. Facultad de Medicina Veterinaria y Zootecnia, Veracruz, México.

d INIFAP. Centro Nacional de Investigación Disciplinaria en Fisiología y Mejoramiento Animal, Querétaro, México.

*Autor de correspondencia: montano.moises@inifap.gob.mx

\section{Resumen:}

El objetivo fue presentar resultados generados en el Instituto Nacional de Investigaciones Forestales, Agrícolas y Pecuarias, sobre la caracterización productiva, genética y molecular del Criollo Coreño (C) de la Sierra Madre Occidental, así como los retos y perspectivas de investigación a corto, mediano y largo plazo. Se evaluaron 35 características de crecimiento, calidad de la canal, fertilidad y producción de leche, en bovinos C, Guzerat (G), CG y GC generados en cruzamiento dialélico, lo que reveló la influencia de la heterosis individual en 
la reproducción y producción de leche, así como de la heterosis materna en los kilogramos de becerro destetado por vaca en empadre. Los efectos genéticos directos y maternos no influyeron de manera relevante sobre las características analizadas. Se observó que toretes $\mathrm{C}$ alimentados con dietas energéticamente altas producen carne con perfiles favorables de ácidos grasos y canales de buena calidad, y que es factible utilizar dosis reducidas de FSH para la superovulación en vaquillas $\mathrm{C}$ sin afectar la producción de embriones. Los resultados señalan a las vacas GC como la mejor opción para producir becerros para engorda, entre los grupos genéticos evaluados. Los resultados muestran también que la población $\mathrm{C}$ mantiene niveles importantes de diversidad genética. Se recomienda, para las poblaciones mexicanas de bovino Criollo, realizar estudios de diversidad genética utilizando información genética molecular e integrar hatos para el registro sistemático de fenotipos relacionados con su productividad y adaptabilidad, y así poder identificar genes exclusivos de interés en esta población.

Palabras clave: Bovinos carne, Caracterización productiva, Criollo Coreño, Cruzamiento dialélico, Diversidad genética, Heterosis.

Recibido: 27/11/2020

Aceptado: 09/03/2021

\section{Introducción}

En México hay varias poblaciones de bovinos criollos, descendientes de los bovinos introducidos durante la Colonia, entre las que se encuentra el Criollo Coreño de la Sierra Madre Occidental. Estas poblaciones se han mantenido aisladas por más de 500 años en diferentes regiones del país, sujetas a la selección natural en condiciones adversas, por la baja disponibilidad de alimento y la ausencia total de manejo sanitario ${ }^{(1)}$. Por ello, se considera que estos animales han desarrollado la capacidad genética para adaptarse a condiciones difíciles, y pueden contribuir a la sustentabilidad de los sistemas vaca-cría al no requerir cambios radicales en los agostaderos que utilizan. La rusticidad de estas poblaciones proviene de la selección natural y se refleja a través de características que les permiten superar las variaciones aleatorias y adversas del medio ambiente, sin disminuir de forma significativa su capacidad productiva. Así, la diversidad genética del bovino Criollo mexicano constituye un recurso genético importante que puede contribuir al diseño de sistemas de producción pecuaria con requerimientos de bajos insumos. En mayor o menor medida, las poblaciones de bovinos criollos han estado disminuyendo o han sufrido erosión genética como resultado del cruzamiento con razas transfronterizas, por lo que cada una de ellas puede estar en una situación de riesgo diferente. 
La conservación exitosa de las poblaciones de bovinos criollos depende del conocimiento que se tenga de las mismas. El objetivo principal de la conservación debe ser preservar tanta diversidad genética como sea posible. La información que es importante conocer para definir una estrategia para la conservación de las poblaciones de bovinos criollos es: 1) el tamaño efectivo de población, que depende del número de machos y hembras que se usan como reproductores cada generación y permite conocer los niveles de consanguinidad y la posible pérdida de diversidad genética; 2) la diversidad genética de la población, que puede determinarse con el conocimiento de la historia de la raza o con su información genética molecular, que puede también usarse para cuantificar el potencial de evolución futura e identificar la existencia de genes asociados a características de interés presente o futuro; 3 ) el comportamiento fenotípico en características de producción y adaptación, que puede utilizarse para estimar su variabilidad genética ${ }^{(2)}$.

Los núcleos de ganado bovino Criollo de la Sierra Madre Occidental denominados Criollo Coreño (C) se localizan en las comunidades indígenas de dicho sistema montañoso, que comparten los estados de Durango, Jalisco, Nayarit y Zacatecas. Tradicionalmente, estas poblaciones se han utilizado para producir becerros al destete en regiones agroecológicas caracterizadas por la escasa disponibilidad de alimento durante el año, y por presentar condiciones ambientales difíciles que se reflejan en la disminución de la eficiencia productiva del ganado C. Se estima que existen alrededor de 16,000 cabezas de ganado C en esta región que forma parte del área de influencia del Sitio Experimental "El Verdineño" (S.E. "El Verdineño") del Instituto Nacional de Investigaciones Forestales, Agrícolas y Pecuarias $(\text { INIFAP) })^{(3)}$.

El S.E. "El Verdineño" se ubica en el municipio de Santiago Ixcuintla, Nayarit, México, en las coordenadas $21^{\circ} 42^{\prime} \mathrm{N} 105^{\circ} 07^{\prime} \mathrm{O}$. El clima es tropical cálido subhúmedo Aw 2 , altitud de $60 \mathrm{msnm}$, precipitación pluvial promedio de $1,200 \mathrm{~mm}$ y temperatura media anual de $24{ }^{\circ} \mathrm{C}$. Cuenta con vegetación de selva mediana subcaducifolia en sucesión secundaria y praderas introducidas de Andropogon, Megathyrsus y Cynodon.

En este S.E. se tiene un hato de bovinos $\mathrm{C}$ para fines experimentales, que se originó a partir de una adquisición de 50 vacas y 10 toros, realizada en 1982. Los animales se adquirieron en comunidades rurales de la Sierra Madre Occidental y no se contaba con ningún tipo de información sobre ellos. Para 1990 ya se contaba con un hato de 70 hembras nacidas en el S.E. "El Verdineño", de las cuales se tenía información genealógica y productiva y de las que se conocían las condiciones de manejo a las que habían estado sujetas. Con estos animales se inició un cruzamiento dialélico con animales Guzerat $(\mathrm{G})$ para evaluar la capacidad productiva y estimar los efectos genéticos directos y maternos del C (Bos taurus) y del G (Bos indicus) y los de heterosis individual y materna, para características de importancia económica ${ }^{(4)}$. En la primera etapa del cruzamiento dialélico (1990-1994) se utilizaron 14 toros $\mathrm{C}$ y 12 toros $\mathrm{G}$ que se aparearon con 70 vacas $\mathrm{C}$ y 70 vacas $\mathrm{G}$, para producir 
animales puros C y G y sus cruzas recíprocas F1, CG y GC. A partir de 1993, en la segunda etapa del cruzamiento dialélico, las hembras de los cuatro grupos raciales (C, G, CG y GC), producidas en la primera etapa, se aparearon con toros Angus Rojo. En ambas etapas las crías permanecieron con las vacas hasta el destete, que se realizó a los siete meses de edad, en promedio.

El objetivo es presentar los resultados generados por el INIFAP sobre la caracterización productiva, genética y molecular del bovino $\mathrm{C}$ de la Sierra Madre Occidental a través de la caracterización de los grupos raciales de las vacas producidas en el cruzamiento dialélico, así como plantear los retos y perspectivas de investigación a corto, mediano y largo plazo para las poblaciones de bovinos criollos de México.

\section{Caracterización productiva del bovino Criollo Coreño}

Estudios realizados en el INIFAP han demostrado que el ganado C puede contribuir a mejorar la producción de becerros para engorda en la región tropical cálida subhúmeda del Occidente de México ${ }^{(5,6,7)}$. Además, se ha documentado que toretes $\mathrm{C}$ y sus cruzas con $\mathrm{G}$ o Angus Rojo, desarrollados y finalizados en confinamiento en la región tropical de Nayarit, producen canales de buena calidad y con buenos rendimientos ${ }^{(8,9)}$. A continuación, se presentan resultados sobre la caracterización productiva, genética y molecular del ganado C durante el período 1990-2019.

\section{Efectos raciales, genéticos aditivos y genéticos no aditivos}

En el Cuadro 1 se presentan las medias de cuadrados mínimos para características reproductivas, de crecimiento predestete, de producción y calidad de leche, de crecimiento en corral y de la canal de bovinos C, G, GC y CG, mientras que en el Cuadro 2 se muestran los estimadores de efectos genéticos directos $\left(\mathrm{g}_{\mathrm{i}}\right)$, maternos $\left(\mathrm{g}_{\mathrm{m}}\right)$, de abuela $\left(\mathrm{g}_{\mathrm{n}}\right)$ y de heterosis individual $\left(\mathrm{h}_{\mathrm{i}}\right)$ y materna $\left(\mathrm{h}_{\mathrm{m}}\right)$ para las mismas características.

Cuadro 1: Medias de cuadrados mínimos para características reproductivas, de crecimiento predestete, de producción y calidad de leche, de crecimiento en corral y de la canal de bovinos Guzerat (G), Guzerat x Criollo Coreño (GC), Criollo Coreño x Guzerat (CG) y Criollo Coreño (C), publicadas en el periodo 2006-2012

\begin{tabular}{lrrrr}
\hline & \multicolumn{4}{c}{ Grupo racial } \\
\cline { 2 - 5 } Característica & G & \multicolumn{1}{c}{ GC } & CG & \multicolumn{1}{c}{ C } \\
\hline${ }^{1}$ Tasa de estro, \% & $49^{\mathrm{a}}$ & $82^{\mathrm{b}}$ & $75^{\mathrm{b}}$ & $69^{\mathrm{b}}$ \\
${ }^{1}$ Tasa de gestación, \% & $46^{\mathrm{a}}$ & $72^{\mathrm{b}}$ & $64^{\mathrm{b}}$ & $60^{\mathrm{b}}$ \\
${ }^{1}$ Tasa de parto, \% & $42^{\mathrm{a}}$ & $71^{\mathrm{b}}$ & $56^{\mathrm{ac}}$ & $59^{\mathrm{bc}}$ \\
${ }^{1}$ Tasa de destete, \% & $38^{\mathrm{a}}$ & $63^{\mathrm{b}}$ & $54^{\mathrm{b}}$ & $52^{\mathrm{b}}$ \\
${ }^{2}$ Peso al nacer, $\mathrm{kg}$ & $31.72^{\mathrm{a}}$ & $30.44^{\mathrm{ab}}$ & $31.11^{\mathrm{a}}$ & $28.86^{\mathrm{b}}$ \\
${ }^{2}$ Peso al destete, kg & $177.42^{\mathrm{a}}$ & $187.45^{\mathrm{a}}$ & $183.34^{\mathrm{a}}$ & $153.50^{\mathrm{b}}$
\end{tabular}


Rev Mex Cienc Pecu 2021;12(Supl 3):23-38

${ }^{2}$ Kilogramos de becerro parido por vaca en

empadre, $\mathrm{kg}$

${ }^{2}$ Kilogramos de becerro destetado por vaca en

empadre, $\mathrm{kg}$

${ }^{2}$ Duración de la gestación, días

${ }^{3}$ Producción total de leche, $\mathrm{kg}$

${ }^{3}$ Producción diaria de leche, $\mathrm{kg}$

${ }^{3}$ Producción máxima en lactancia, kg

${ }^{3}$ Día de máxima producción, días

${ }^{3}$ Persistencia de la lactancia, días

${ }^{4}$ Grasa láctea, \%

${ }^{4}$ Grasa láctea, $\mathrm{kg}$

${ }^{4}$ Proteína láctea, \%

${ }^{4}$ Proteína láctea, $\mathrm{kg}$

${ }^{4}$ Lactosa, \%

${ }^{4}$ Lactosa, kg

${ }^{4}$ Sólidos no grasos lácteos, \%

${ }^{4}$ Sólidos no grasos lácteos, $\mathrm{kg}$

${ }^{5}$ Peso al inicio de la engorda en corral, $\mathrm{kg}$

${ }^{5}$ Peso al año, kg

${ }^{5}$ Peso al final de la engorda en corral, $\mathrm{kg}$

${ }^{5}$ Ganancia diaria de peso en corral, $\mathrm{kg}$

${ }^{5}$ Eficiencia alimenticia en corral, $\mathrm{kg}$

${ }^{5}$ Área del ojo de la costilla, pulgadas

${ }^{5}$ Grasa de cobertura, pulgadas

${ }^{5}$ Peso de canal caliente, $\mathrm{kg}$

${ }^{5}$ Porcentaje de rendimiento en canal, \%

${ }^{5}$ Grasa en riñón y pelvis, $\mathrm{kg}$

${ }^{5}$ Grado de rendimiento de canal, unidades

${ }^{5}$ Porcentaje de rendimiento en cortes, $\%$ $17.69^{\mathrm{a}}$

$98.44^{\mathrm{ac}}$

$283.25^{\mathrm{ab}}$

$949^{\mathrm{a}}$

$4.50^{\mathrm{a}}$

$6.50^{\mathrm{a}}$

$76^{\mathrm{a}}$

$147^{\mathrm{a}}$

2.80

25.90

$3.56^{\mathrm{a}}$

33.90

4.79

$47.10^{\mathrm{a}}$

9.09

$88.20^{\mathrm{a}}$

$226.00^{\mathrm{a}}$

$359.50^{\mathrm{a}}$

$405.60^{\mathrm{a}}$

$1.15^{\mathrm{a}}$

$0.105^{\mathrm{a}}$

$10.73^{\mathrm{a}}$

$0.37^{\mathrm{a}}$

$241.70^{\mathrm{a}}$

$60.20^{\mathrm{a}}$

$8.06^{\mathrm{a}}$

$2.61^{\mathrm{a}}$

$50.79^{\mathrm{a}}$

$76.58^{\mathrm{a}}$ $19.73^{\mathrm{a}}$

$18.21^{\mathrm{a}}$

$16.38^{\mathrm{a}}$

${ }^{5}$ Porcentaje total de cortes al menudeo, \%

$143.50^{\mathrm{b}} \quad 116.15^{\mathrm{ab}} \quad 83.97^{\mathrm{c}}$

$283.87^{\mathrm{ab}} \quad 280.98^{\mathrm{a}} \quad 284.10^{\mathrm{b}}$

$1,059^{\mathrm{b}} \quad 990^{\mathrm{ab}} \quad 805^{\mathrm{c}}$

$\begin{array}{lll}5.00^{\mathrm{b}} & 4.70^{\mathrm{ab}} & 3.90^{\mathrm{c}}\end{array}$

$\begin{array}{lll}7.30^{\mathrm{b}} & 6.70^{\mathrm{ab}} & 5.80^{\mathrm{c}}\end{array}$

$147^{\mathrm{a}} \quad 149^{\mathrm{a}} \quad 143^{\mathrm{a}}$

$\begin{array}{lll}2.98 & 3.09 & 2.81\end{array}$

$\begin{array}{lll}29.50 & 28.80 & 22.30\end{array}$

$\begin{array}{lll}3.83^{\mathrm{b}} & 3.72^{\mathrm{b}} & 3.88^{\mathrm{b}}\end{array}$

$\begin{array}{lll}38.20 & 37.20 & 31.60\end{array}$

$\begin{array}{lll}4.82 & 4.75 & 4.71\end{array}$

$52.10^{\mathrm{a}} \quad 48.60^{\mathrm{a}} \quad 39.50^{\mathrm{b}}$

$\begin{array}{lrr}9.36 & 9.19 & 9.29\end{array}$

$\begin{array}{lll}98.90^{\mathrm{a}} & 93.20^{\mathrm{a}} & 76.90^{\mathrm{b}}\end{array}$

$206.70^{\mathrm{ab}} \quad 208.90^{\mathrm{b}} \quad 164.60^{\mathrm{c}}$

$338.50^{\mathrm{ab}} \quad 329.50^{\mathrm{b}} \quad 279.40^{\mathrm{c}}$

$388.60^{\mathrm{ab}} \quad 377.10^{\mathrm{b}} \quad 335.20^{\mathrm{c}}$

$\begin{array}{lll}1.13^{\mathrm{a}} & 1.07^{\mathrm{a}} & 1.09^{\mathrm{a}}\end{array}$

$\begin{array}{lll}0.114^{\mathrm{b}} & 0.103^{\mathrm{a}} & 0.109^{\mathrm{ab}}\end{array}$

$\begin{array}{lll}11.83^{\mathrm{a}} & 9.66^{\mathrm{b}} & 8.40^{\mathrm{c}}\end{array}$

$\begin{array}{lll}0.48^{\mathrm{a}} & 0.38^{\mathrm{a}} & 0.40^{\mathrm{a}}\end{array}$

$249.60^{\mathrm{a}} \quad 210.60^{\mathrm{b}} \quad 187.50^{\mathrm{b}}$

$58.60^{\mathrm{ab}} \quad 58.20^{\mathrm{b}} \quad 54.20^{\mathrm{c}}$

$2.91^{\mathrm{b}} \quad 5.34^{\mathrm{b}} \quad 7.95^{\mathrm{a}}$

$2.92^{\mathrm{ab}} \quad 2.62^{\mathrm{a}} \quad 3.21^{\mathrm{b}}$

$50.06^{\mathrm{ab}} \quad 50.80^{\mathrm{a}} \quad 49.48^{\mathrm{b}}$

$75.12^{\mathrm{ab}} \quad 76.61^{\mathrm{a}} \quad 73.97^{\mathrm{b}}$ $74^{\mathrm{a}} \quad 77^{\mathrm{a}} \quad 70^{\mathrm{a}}$

Fuente: ${ }^{1} \mathrm{Martinez}$ et $a l^{(5)}$. ${ }^{2} \mathrm{Martinez}$ et $a l^{(6)}$. ${ }^{3} \mathrm{Martinez}$ et $a l^{(11)}$. ${ }^{4} \mathrm{Martinez}$ et $a l^{(12) .}{ }^{5} \mathrm{Martinez}$ et al ${ }^{(8)}$

\section{Comportamiento reproductivo de hembras y crecimiento predestete de sus crías}

En un estudio en el que se evaluaron características reproductivas, hembras C, CG y GC mostraron mayores $(P<0.10)$ tasas de estro $(\mathrm{TE})$ y gestación $(\mathrm{TG})$ que hembras $\mathrm{G}$, con diferencias de 20, 26 y 33 puntos porcentuales en TE, y de 14, 18 y 26 puntos porcentuales en TG. Esto indica que las hembras C, CG y GC son reproductivamente más eficientes. Sin embargo, no se observaron diferencias entre G y CG en la tasa de parto (TP) (Cuadro 1), lo que pudo deberse a una mayor pérdida embrionaria o fetal en las hembras CG, sugiriendo que las hembras $\mathrm{G}$ proveen un ambiente uterino menos favorable para la sobrevivencia del embrión o del feto que las hembras C. En forma similar, la TP sugiere un ambiente uterino desfavorable de las hembras CG para la sobrevivencia prenatal de sus crías, comparado con el de las GC. Las vacas $\mathrm{C}, \mathrm{CG}$ y GC tuvieron mayor $(P<0.10)$ tasa de destete (TD) que las $\mathrm{G}$ (14, 16 y 25 puntos porcentuales más, respectivamente; Cuadro 1); en relación con esto, los 
autores señalaron que se pudo deber a una menor habilidad materna de las vacas G durante el periodo nacimiento-destete, una menor sobrevivencia de sus crías durante este periodo, o la combinación de ambas desventajas. Los estimadores de $\mathrm{h}_{\mathrm{i}}$ indicaron que ésta influyó $(P<0.10)$ de manera favorable sobre todas las tasas reproductivas, lo que se manifestó en una mayor $(P<0.10)$ TD de las hembras cruzadas con respecto a la de las puras; por el contrario, no se observaron diferencias importantes entre los $\mathrm{g}_{\mathrm{i}} \mathrm{de} \mathrm{C}$ y $\mathrm{G}$ para ninguna variable reproductiva (Cuadro 2). Igualmente, la diferencia entre los $\mathrm{g}_{\mathrm{m}}$ indica que ésta fue poco importante para TE, TG y TD; sin embargo, sí se observaron diferencias $(P<0.10)$ entre los $\mathrm{g}_{\mathrm{m}}$ para TP a favor de las vacas C (15 puntos porcentuales más) (Cuadro 2$)^{(5)}$.

Cuadro 2: Estimadores de efectos genéticos directos $\left(\mathrm{g}_{\mathrm{i}}\right)$, maternos $\left(\mathrm{g}_{\mathrm{m}}\right)$, de abuela $\left(\mathrm{g}_{\mathrm{n}}\right)$ y de heterosis individual $\left(\mathrm{h}_{\mathrm{i}}\right)$ y materna $\left(\mathrm{h}_{\mathrm{m}}\right)$ para características reproductivas, de crecimiento predestete, de producción y calidad de leche, de crecimiento en corral y de la canal, estimados a partir de un dialelo generado con bovinos Criollo Coreño (Bos taurus) y

Guzerat (Bos indicus) y publicados en el periodo 2006-2012

\begin{tabular}{|c|c|c|c|c|c|}
\hline \multirow[b]{2}{*}{ Característica } & \multicolumn{5}{|c|}{ Efecto genético } \\
\hline & $\mathbf{h}_{\mathrm{i}}$ & $\mathbf{h}_{\mathrm{m}}$ & $g_{i}$ & $g_{m}$ & $\mathbf{g}_{\mathbf{n}}$ \\
\hline${ }^{1}$ Tasa de estro, $\%$ & $20.00^{\beta}$ & & -14.00 & -6.00 & \\
\hline${ }^{1}$ Tasa de gestación, $\%$ & $15.00^{\beta}$ & & -6.00 & -7.00 & \\
\hline${ }^{1}$ Tasa de parto, $\%$ & $13.00^{\beta}$ & & -2.00 & & \\
\hline & & & & $15.0^{\beta}$ & \\
\hline${ }^{1}$ Tasa de destete, $\%$ & $13.00^{\beta}$ & & -5.00 & -9.00 & \\
\hline${ }^{2,4} \mathrm{Peso}$ al nacer, $\mathrm{kg}$ & & 0.48 & $3.80 *$ & 0.74 & \\
\hline 2,4 Peso al destete, $\mathrm{kg}$ & & 19.93* & $37.60 *$ & 8.45 & \\
\hline${ }^{2}$ Kilogramos de becerro parido por vaca en empadre, $\mathrm{kg}$ & & 1.93 & & & \\
\hline $\begin{array}{l}{ }^{2} \text { Kilogramos de becerro destetado por vaca en empadre, } \\
\mathrm{kg}\end{array}$ & & $38.62 *$ & & & \\
\hline${ }^{2}$ Duración de la gestación, días & -1.25 & & & & \\
\hline${ }^{3}$ Producción total de leche, $\mathrm{kg}$ & $147.00 *$ & & & & \\
\hline${ }^{3}$ Producción diaria de leche, $\mathrm{kg}$ & $0.60 *$ & & & & \\
\hline${ }^{3}$ Producción máxima en lactancia, kg & $0.80 *$ & & & & \\
\hline ºía de máxima producción, días & 2.50 & & & & \\
\hline Persistencia de la lactancia, días & 3.00 & & & & \\
\hline${ }^{4}$ Grasa láctea, \% & 0.23 & & -0.12 & -0.11 & \\
\hline${ }^{4}$ Grasa láctea, kg & $5.07 *$ & & 4.34 & 0.68 & \\
\hline${ }^{4}$ Proteína láctea, $\%$ & 0.05 & & $-0.21 *$ & 0.11 & \\
\hline${ }^{4}$ Proteína láctea, $\mathrm{kg}$ & $4.97 *$ & & 3.38 & 1.06 & \\
\hline${ }^{4}$ Lactosa, \% & 0.03 & & 0.15 & 0.07 & \\
\hline 'Lactosa, kg & 7.04* & & 11.08* & 3.48 & \\
\hline${ }^{4}$ Sólidos no grasos lácteos, \% & 0.09 & & -0.03 & 0.17 & \\
\hline${ }^{4}$ Sólidos no grasos lácteos, kg & 13.48* & & $16.98 *$ & 6.64 & \\
\hline${ }^{5}$ Peso al inicio de la engorda en corral, $\mathrm{kg}$ & & 12.51 & & & 2.26 \\
\hline${ }^{5}$ Peso al año, kg & & 14.60 & & & -9.05 \\
\hline${ }^{5}$ Peso al final de la engorda en corral, $\mathrm{kg}$ & & 12.40 & & & -11.47 \\
\hline${ }^{5}$ Ganancia diaria de peso en corral, $\mathrm{kg}$ & & -0.02 & & & -0.06 \\
\hline${ }^{5}$ Eficiencia alimenticia en corral, $\mathrm{kg}$ & & 0.02 & & & $-0.01^{\beta}$ \\
\hline${ }^{5}$ Área del ojo de la costilla, pulgadas & & $1.18^{\beta}$ & & & $-2.20^{\beta}$ \\
\hline${ }^{5}$ Grasa de cobertura, pulgadas & & 0.04 & & & \\
\hline${ }^{5}$ Peso de canal caliente, $\mathrm{kg}$ & & 15.5 & & & $-39.02^{\beta}$ \\
\hline${ }^{5}$ Porcentaje de rendimiento en canal, $\%$ & & 1.16 & & & \\
\hline
\end{tabular}


${ }^{5} \mathrm{Grasa}$ en riñón y pelvis, $\mathrm{kg}$

$-\mathbf{- 3 . 8 8}^{\beta}$

${ }^{5}$ Grado de rendimiento de canal, unidades

$-0.14$

${ }^{5}$ Porcentaje de rendimiento en cortes, $\%$

0.30

${ }^{5}$ Porcentaje total de cortes al menudeo, $\%$

0.59

Efecto genético directo $=$ Guzerat-Criollo.

Efecto genético materno= Criollo-Guzerat.

Efectos genéticos en negritas fueron estadísticamente significativos: $*(P<0.05) ;{ }^{\beta}(P<0.10)$.

Fuente: ${ }^{1}$ Martínez et $a l^{(5)}$; ${ }^{2}$ Martínez et $a l^{(6)} ;{ }^{3} \mathrm{Martinez} \mathrm{et} a l^{(11)} ;{ }^{4} \mathrm{Martínez} \mathrm{et} a l^{(12) ;}{ }^{5} \mathrm{Martínez} \mathrm{et} a l^{(8)}$.

Por otro lado, para estudiar algunos aspectos relacionados con la superovulación de hembras $\mathrm{C}$, se realizó un estudio en el que se aplicaron dosis reducidas de FSH en tres tratamientos $(\mathrm{T} 1=280 \mathrm{mg}, \mathrm{T} 2=200 \mathrm{mg}$ y $\mathrm{T} 3=140 \mathrm{mg}$ ) a vacas (experimento 1 ) y vaquillas $\mathrm{C}$ (experimento $2)$, encontrándose diferencias $(P<0.05)$ entre tratamientos para número de corpúsculos recuperados (CR) y número de óvulos no fertilizados (ONF) favorables a T1; sin embargo, no se detectaron diferencias importantes entre tratamientos para número de embriones transferibles (ET), número de embriones no transferibles (ENT), número de cuerpos lúteos (CL), volumen ovárico (VO), concentración sérica de progesterona (P4), porcentaje de fertilización $(\% \mathrm{~F})$ y porcentaje de recuperación $(\% \mathrm{R})$, por lo que los autores comentaron que los promedios de ENT, CL, ONF, VO y \%R señalaron a T1 como el mejor tratamiento para favorecer la superovulación en vacas. Además, en éstas se observó una reducción en CR a medida que disminuyó la dosis de FSH, observándose la mayor respuesta con T1 (10.3 corpúsculos recuperados), que fue diferente $(P<0.05)$ a la observada con T3 $(4.1$ corpúsculos recuperados); en contraste, se encontró que $\mathrm{T} 1$ produjo el mayor $\mathrm{ONF}$, que fue diferente $(P<0.05)$ al producido con T3. Lo anterior mostró que T3 promovió una menor respuesta superovulatoria en vacas, comparado con T1; sin embargo, con T1 no se tuvo una mayor producción de embriones, debido al gran ONF producido con este tratamiento. Por el contrario, no se detectaron diferencias estadísticas entre tratamientos para ninguna variable en vaquillas. La similitud entre tratamientos mostró la factibilidad de utilizar dosis reducidas de FSH en vaquillas sin afectar su respuesta a la superovulación. Para el total de hembras se encontró una correlación positiva $(P<0.05)$ entre CL y P4, lo que se reflejó en que las hembras con mayor CL fueron las que presentaron las mayores P4. Además, se detectó una correlación positiva $(P<0.05)$ entre VO y $\mathrm{P} 4$, lo que significó que las hembras con mayor VO presentaron también la mayor P4. Considerando los resultados de los tres tratamientos en ambos experimentos, se observó que las vacas respondieron mejor a la superovulación al producir mayor CL (10.4, 9.1 y 8.8 para T1, T2 y T3, respectivamente) que las vaquillas (5.8, 5.5 y 4.5 para T1, T2 y T3, respectivamente); en contraste, las vacas tuvieron menor \%F (44.6, 52.5 y $36.9 \%$ para T1, T2 y T3, respectivamente) que las vaquillas $(96.5,95.7$ y $97.3 \%$ para T1, T2 y T3, respectivamente). Igualmente, considerando los resultados de los tres tratamientos en ambos experimentos, se observó que las vacas tuvieron mayor VO, CL y P4 que las vaquillas. Con los tres tratamientos en ambos experimentos, se observó que la respuesta ovulatoria fue menor en vaquillas $(3.8,2.3$ y 2.0 corpúsculos recuperados para T1, T2 y T3, respectivamente) que en vacas $(10.3,7.2$ y 4.0 corpúsculos recuperados para T1, T2 y T3, respectivamente); sin embargo, el ET (2.3, 1.8 y 1.7 para T1, T2 y T3, respectivamente) 
y el ENT (1.3, 0.3 y 0.2 para T1, T2 y T3, respectivamente) en vaquillas fueron similares al ET (1.2, 2.9 y 1.1 para T1, T2 y T3, respectivamente) y ENT (2.0, 1.8 y 0.8 para T1, T2 y T3, respectivamente) en vacas. Los promedios de ET y ENT de vacas y vaquillas indicaron que vacas y vaquillas tuvieron producciones embrionarias similares, debido a una mayor fertilidad de las vaquillas. No se detectaron diferencias importantes entre tratamientos en el ET en ninguno de los dos experimentos. Finalmente, los autores concluyeron que es factible utilizar dosis reducidas de FSH para la superovulación en vaquillas C, disminuyendo así los costos de superovulación y producción de embriones. Las vacas $\mathrm{C}$ mostraron una mejor respuesta a la superovulación comparadas con las vaquillas; en contraste, las vaquillas tuvieron mayor $\% \mathrm{~F}^{(10)}$.

En un estudio en el que se analizaron características productivas predestete, las crías de vacas G y CG tuvieron mayores $(P<0.05)$ pesos al nacer $(\mathrm{PN})$ que las de vacas $\mathrm{C}$, con una diferencia de hasta $2.9 \mathrm{~kg}$; por su parte, las crías de vacas GC mostraron PN intermedios (Cuadro 1). Las crías de vacas G, CG y GC tuvieron mayores $(P<0.05)$ pesos al destete $(\mathrm{PD})$ que las de vacas $C$, con una diferencia de 24, 30 y $34 \mathrm{~kg}$, respectivamente; en contraste, no se detectaron diferencias $(P>0.05)$ en los kilogramos de becerro parido por vaca en empadre (KBP) entre estos grupos raciales (Cuadro 1$)$. Las vacas GC produjeron más $(P<0.05)$ kilogramos de becerro destetado por vaca en empadre (KBD) que las $\mathrm{G} \mathrm{y} \mathrm{C}$, mientras que las $\mathrm{CG}$ solo fueron superiores $(P<0.05)$ a las $\mathrm{C}$ (Cuadro 1). Las hembras $\mathrm{C}$ tuvieron una gestación $3.1 \mathrm{~d}$ más larga $(P<0.05)$ que las $\mathrm{CG}$, mientras que las $\mathrm{GC}$ y $\mathrm{G}$ mostraron una duración de la gestación (DG) intermedia (Cuadro 1$)$. La $h_{i}$ no fue importante para DG $(P>0.05)$, ni la $h_{m}$ para PN y KBP $(P>0.05)$; en contraste, la $\mathrm{h}_{\mathrm{m}}$ fue importante $(P<0.05)$ para PD y KBD (Cuadro 2$)$. En relación a $\mathrm{PD}$, el efecto de la $\mathrm{h}_{\mathrm{m}}$ se reflejó en un incremento de $19.93 \mathrm{~kg}(P<0.05)$; por su parte, el estimador de $\mathrm{h}_{\mathrm{m}}$ para KBD indica que, en promedio, las vacas GC y CG produjeron 38.6 kilogramos más de becerro al destete $(P<0.05)$ que las $\mathrm{G}$ y $\mathrm{C}$ (Cuadro 2$)$. Las vacas $\mathrm{GC}$ produjeron entre 45 y 60 más KBD que las vacas $\mathrm{G}$ o $\mathrm{C}(P<0.05)$, lo que señala a este grupo genético como una opción recomendable para aumentar la productividad al destete en hatos $\mathrm{G}$ o $\mathrm{C}$ que producen becerros para la engorda ${ }^{(6)}$.

\section{Producción y calidad de leche}

Por otro lado, en una investigación en la que se analizó la producción de leche y su relación con $\mathrm{PD}$, se encontró que las vacas $\mathrm{GC}$ tuvieron mayor $(P<0.10)$ producción total de leche (PTL; 1,059 vs 805, 949 y $990 \mathrm{~kg}$, respectivamente), producción diaria de leche (PDL; 5.0 vs 3.9 y $4.5 \mathrm{~kg}$, respectivamente) y producción máxima en lactancia (PML; 7.3 vs 5.8 y 6.5 $\mathrm{kg}$, respectivamente) que las $\mathrm{C} \mathrm{y} \mathrm{G}$, mientras que las $\mathrm{CG}$ tuvieron un desempeño intermedio. Entre G y C también se encontraron diferencias $(P<0.10)$ favorables a $\mathrm{G}$ en PTL $(144 \mathrm{~kg})$ y PDL $(0.6 \mathrm{~kg})$. Por el contrario, no se observaron diferencias en día de máxima producción (DMP) y persistencia de la lactancia (PER) entre grupos genéticos (Cuadro 1). La hi influyó 
$(P<0.05)$ sobre PTL, PDL y PML, pero no influyó sobre DMP y PER. Igualmente, se detectaron diferencias $(P<0.05)$ en $\mathrm{g}_{\mathrm{i}}$ favorables a $\mathrm{G}$ sobre $\mathrm{C}$ para PTL, PDL y PML; por el contrario, no se observaron diferencias en $\mathrm{g}_{\mathrm{m}}$ para ninguna variable de producción de leche (Cuadro 2). Los estimadores de la correlación entre PTL y PD fueron significativos $(P<0.05)$ para todos los grupos genéticos; sin embargo, la diferencia entre los estimadores para las cruzas recíprocas indicó una correlación más fuerte para $\mathrm{GC}(\mathrm{r}=0.49)$ que para $\mathrm{CG}(\mathrm{r}=0.25)$. En relación con estos estimadores, los autores argumentaron que la recomendación de utilizar vacas GC o CG para producción de becerros al destete, debe incluir la utilización de estrategias diferentes para el manejo alimenticio durante la lactancia de cada uno de estos grupos genéticos. El estimador de la correlación global entre PTL y PD para los cuatro grupos genéticos fue $0.44(P<0.05)$. Los mayores coeficientes de regresión de PD sobre PTL correspondieron a $\mathrm{C}$ y GC $(P<0.05)$, por lo que los autores comentaron que estos coeficientes sugieren que crías de madres o abuelas $C$ tuvieron una menor dependencia nutricional de leche materna y dependieron en gran medida de fuentes alimenticias alternas de menor valor nutricional. En relación a los kilogramos de leche por lactancia requeridos por cada grupo genético para producir $1 \mathrm{~kg}$ de $\mathrm{PD}$, los autores comentaron que dichos resultados sugieren un uso más eficiente de la leche disponible por parte de las crías de vacas $\mathrm{C}$ y GC, que consumieron, en promedio, 22.5 y $27.3 \mathrm{~kg}$ de leche por cada kilogramo de PD, mientras que el consumo de las crías de vacas G y CG fue de 30.9 y $37.3 \mathrm{~kg}$, respectivamente. Además, hicieron hincapié en que la diferencia de $10 \mathrm{~kg}$ de leche consumida entre crías de vacas CG y GC, para producir $1 \mathrm{~kg}$ de PD, debe tomarse en cuenta al hacer recomendaciones sobre el uso de estos grupos genéticos para la producción de becerros al destete ${ }^{(11)}$.

En un estudio complementario al de producción de leche, en el que se determinó la composición de la misma y su relación con PD, no se observaron diferencias en porcentaje de grasa (PG), lactosa (PL) y sólidos no grasos (PS), ni en kilogramos de grasa (KG) y proteína (KP), entre grupos genéticos; en contraste, la leche de vacas $\mathrm{C}, \mathrm{GC}$ y CG tuvo mayor $(P<0.05)$ porcentaje de proteína $(\mathrm{PP})$ que la de vacas $\mathrm{G}$; además, las vacas $\mathrm{G}, \mathrm{GC}$ y CG produjeron más $(P<0.05)$ kilogramos de lactosa $(\mathrm{KL})$ y sólidos no grasos $(\mathrm{KS})$ que las $\mathrm{C}$ (Cuadro 1). Las vacas $\mathrm{G}$ parieron becerros más pesados $(P<0.05)$ que las $\mathrm{C}$, $\mathrm{GC}$ y $\mathrm{CG}$, mientras que las vacas $\mathrm{G}, \mathrm{GC}$ y $\mathrm{CG}$ destetaron becerros más pesados $(P<0.05)$ que las $\mathrm{C}$ (Cuadro 1). El hecho de que los becerros de vacas cruzadas pesaron menos al nacer que los de vacas $\mathrm{G}$ y que los tres grupos genéticos produjeron becerros con PD similares, se atribuyó a un mejor comportamiento productivo durante el período nacimiento-destete de las vacas cruzadas y sus crías. En cuanto a efectos genéticos no aditivos, se encontró que la $\mathrm{h}_{\mathrm{i}}$ no influyó los componentes de leche en porcentaje, pero si influyó $(P<0.05)$ en la expresión de los componentes de leche en kilogramos; además, la $\mathrm{h}_{\mathrm{m}}$ influyó $(P<0.05)$ en la expresión de PN y PD. Los efectos de $\mathrm{h}_{\mathrm{m}}$ fueron importantes $(P<0.05)$ y favorables al disminuir $\mathrm{PN}\left(\mathrm{h}_{\mathrm{m}}=\right.$ $-1.3 \mathrm{~kg})$ y aumentar PD $\left(\mathrm{h}_{\mathrm{m}}=12.7 \mathrm{~kg}\right)$ (Cuadro 2$)$. En lo que concernió a efectos genéticos aditivos, las diferencias entre los $\mathrm{g}_{\mathrm{m}}$ no fueron significativas para ninguna de las variables de composición de leche; sin embargo, las diferencias $(P<0.05)$ entre los $g_{i}$ para PP fueron 
favorables a $\mathrm{C}$ en 0.20 puntos porcentuales. Para componentes de leche en kilogramos las diferencias $(P<0.05)$ entre los $\mathrm{g}_{\mathrm{i}}$ para lactosa y sólidos no grasos fueron favorables para $\mathrm{G}$ en 11.1 y $17.0 \mathrm{~kg}$; igualmente, las diferencias $(P<0.05)$ entre los gi para PN y PD favorecieron a $\mathrm{G}$ en 3.8 y $37.6 \mathrm{~kg}$ (Cuadro 2). Las correlaciones entre PD y los componentes de leche en porcentaje no fueron significativas, excepto para $\mathrm{PP}(\mathrm{r}=-0.18 ; \mathrm{P}<0.05)$, lo que sugiere que la calidad de la leche influyó poco sobre PD. Por otro lado, para componentes de leche en kilogramos se detectaron correlaciones importantes $(P<0.05)$ de PD con KG $(\mathrm{r}=0.16), \mathrm{KL}$ $(\mathrm{r}=0.21)$ y KS $(\mathrm{r}=0.19)$, sin que fuera significativa la correlación con $\mathrm{KP}(\mathrm{r}=0.13)^{(12)}$.

\section{Comportamiento en corral y características de canal}

En un estudio adicional en el que se evalúo el comportamiento en engorda confinada y características de la canal, los pesos al inicio de engorda (PI), año de edad (PA) y final de engorda $(\mathrm{PF})$ de la progenie de vacas $\mathrm{C}$ fueron menores $(P<0.10)$ que los pesos de la progenie de vacas G, GC y CG, con diferencias de hasta $61.4,80.1$ y $70.4 \mathrm{~kg}$ a favor de las vacas G; sin embargo, la ganancia diaria de peso (GDP) y la eficiencia alimenticia (EA) de la progenie de vacas $\mathrm{C}$ fueron similares $(P>0.05)$ a las de la progenie de vacas $\mathrm{G}, \mathrm{GC}$ y $\mathrm{CG}$ (Cuadro 1$)$. La progenie de vacas G y GC tuvo mayor área del ojo de la costilla (AOC) y peso de canal caliente (PCC) que la de vacas CG y C $(P<0.10)$; además, se encontró que la progenie de vacas $\mathrm{C}$ tuvo el menor porcentaje $(P<0.10)$ de rendimiento en canal $(\mathrm{PRC})$, pero similar grasa de cobertura (GRC) en comparación con la progenie de las vacas de los otros grupos genéticos (Cuadro 1). Con relación al grado de rendimiento de la canal (GR), al porcentaje de rendimiento en cortes (RC) y al porcentaje total de cortes al menudeo (PTC), se observó que la progenie de vacas $\mathrm{G}$ y $\mathrm{CG}$ fue superior $(P<0.10)$ a la de vacas $\mathrm{C}$, mientras que las crías de vacas GC tuvieron un desempeño intermedio; sin embargo, $\mathrm{G}$ y $\mathrm{C}$ tuvieron similar cantidad de grasa en riñón y pelvis (RP) (Cuadro 1). Por el otro lado, hijas de vacas $\mathrm{C}$ produjeron becerros con mayor $(P<0.10)$ EA $(0.01 \mathrm{~kg})$ que hijas de vacas G. Similarmente, los nietos de abuelas $\mathrm{C}$ fueron superiores $(P<0.10)$ a los de abuelas $\mathrm{G}$ en AOC $\left(2.2\right.$ pulgadas $\left.^{2}\right)$ y PCC $(39.0 \mathrm{~kg})$. Globalmente, las diferencias tendieron a favorecer a los nietos de abuelas $\mathrm{C}$, aunque sólo se detectaron diferencias significativas $(P<0.10)$ para EA, AOC y PCC. Finalmente, se observó que la $\mathrm{h}_{\mathrm{m}}$ no fue importante $(P>0.10)$ para ninguna de las variables medidas en confinamiento, pero si lo fue $(P<0.10)$ para AOC $\left(1.18\right.$ pulgadas $\left.^{2}\right)$ y RP $(-3.88$ kg) $(\text { Cuadro 2) })^{(8)}$.

Otro estudio ${ }^{(13)}$, cuyo objetivo fue conocer el perfil de ácidos grasos de la carne de toretes $\mathrm{C}$ alimentados con diferentes niveles de energía (NE) en la dieta (NE 1=2.2, NE 2=2.4, NE $3=2.2+2.4$, NE 4=2.4+2.6 Mcal de EM/kg de MS), mostró que la dieta con el mayor nivel de energía (NE 4) resultó en un menor contenido $(P<0.05)$ de ácidos grasos saturados (AGS), pero en un mayor contenido $(P<0.05)$ de ácidos grasos no identificados (Cuadro 3). El contenido de los ácidos palmítico C16.0 y esteárico C18.0 se redujo $(P<0.05)$ al incrementar 
el nivel de energía en la dieta. Los ácidos grasos monoinsaturados (AGM) aumentaron $(P<0.05)$ a partir del NE 2, en contraste, los cambios en el ácido palmitoleico C16:1cis-9 fueron en sentido inverso, pero la cantidad del ácido oleico C18:1cis-9 no varió significativamente (Cuadro 3). Además, el mayor contenido $(P<0.05)$ de ácidos grasos poliinsaturados (AGP) se obtuvo con el mayor contenido de energía (NE 4). Aunque los cambios fueron variables en los ácidos linoleico C18:2 cis-9, 12 (C18:2 $\omega-6)$ y linolénico C18:3 cis-9, 12, $15(\mathrm{C} 18: 3 \omega-3)$, se observaron cambios $(P<0.05)$ por efecto de la energía. Asimismo, las relaciones AGP/AGS, (AGM+AGP)/AGS y C18:2 $\omega-6 / C 18: 3 \omega-3$ fueron afectadas $(P<0.05)$ por la dieta, mejorando con los mayores niveles de energía (Cuadro 3$)$. Estos resultados son de gran importancia en la salud humana, dado que la relación omega6:omega-3 llega hasta 17:1 en algunas dietas occidentales ${ }^{(14)}$.

Cuadro 3: Perfil de ácidos grasos (gramos de ácidos grasos/100 g de grasa) de carne de toretes Criollo Coreño alimentados con distintos niveles de energía

\begin{tabular}{lcccc}
\hline \multicolumn{1}{c}{ Variable } & NE 1 & NE 2 & NE 3 & NE 4 \\
\hline Ácido palmítico C16.0 & $27.0^{\mathrm{a}}$ & $24.2^{\mathrm{c}}$ & $26.6^{\mathrm{a}}$ & $20.5^{\mathrm{b}}$ \\
Ácido palmitoleico C16:1 cis-9 & $3.2^{\mathrm{a}}$ & $3.3^{\mathrm{a}}$ & $3.4^{\mathrm{a}}$ & $2.7^{\mathrm{b}}$ \\
Ácido esteárico C18.0 & $20.7^{\mathrm{a}}$ & $17.6^{\mathrm{b}}$ & $16.9^{\mathrm{b}}$ & $17.0^{\mathrm{b}}$ \\
Ácido oleico C18:1cis-9 & $37.0^{\mathrm{a}}$ & $38.9^{\mathrm{a}}$ & $39.5^{\mathrm{a}}$ & $37.5^{\mathrm{a}}$ \\
Ácido linoleico C18:2 cis-9, 12 & $3.0^{\mathrm{a}}$ & $4.0^{\mathrm{bc}}$ & $3.6^{\mathrm{ac}}$ & $3.8^{\mathrm{ac}}$ \\
Ácido linolénico C18:3 cis-9, 12, 15 & $1.07^{\mathrm{a}}$ & $0.71^{\mathrm{a}}$ & $0.68^{\mathrm{a}}$ & $1.90^{\mathrm{b}}$ \\
Ácidos grasos saturados & $45.0^{\mathrm{a}}$ & $42.8^{\mathrm{a}}$ & $45.0^{\mathrm{a}}$ & $35.0^{\mathrm{b}}$ \\
Ácidos grasos monoinsaturados & $39.7^{\mathrm{ac}}$ & $42.4^{\mathrm{bc}}$ & $42.6^{\mathrm{b}}$ & $41.2^{\mathrm{bc}}$ \\
Ácidos grasos poliinsaturados & $4.1^{\mathrm{b}}$ & $4.6^{\mathrm{b}}$ & $4.6^{\mathrm{b}}$ & $5.5^{\mathrm{a}}$ \\
Relación AGP/AGS & $0.08^{\mathrm{a}}$ & $0.12^{\mathrm{b}}$ & $0.11^{\mathrm{b}}$ & $0.15^{\mathrm{c}}$ \\
Ácidos grasos no identificados & $8.7^{\mathrm{b}}$ & $6.6^{\mathrm{b}}$ & $6.4^{\mathrm{b}}$ & $17.6^{\mathrm{a}}$ \\
Relación (AGP+AGM)/AGS & $0.98^{\mathrm{a}}$ & $1.13^{\mathrm{b}}$ & $1.09^{\mathrm{b}}$ & $1.33^{\mathrm{c}}$ \\
Relación ácido linoleico/ácido linolénico & $0.37^{\mathrm{b}}$ & $0.26^{\mathrm{b}}$ & $0.10^{\mathrm{b}}$ & $0.82^{\mathrm{a}}$ \\
\hline
\end{tabular}

$\mathrm{NE} 1=2.2 \mathrm{Mcal}$ EM/kg MS de inicio a fin; NE 2=2.4 Mcal EM/kg MS de inicio a fin; NE $3=2.2 \mathrm{Mcal}$ $\mathrm{EM} / \mathrm{kg}$ MS del inicio hasta los $300 \mathrm{~kg}$ de peso corporal, continuando con $2.4 \mathrm{Mcal}$ EM/kg MS hasta el final de la prueba; NE 4=2.4 Mcal EM/kg MS del inicio hasta los $300 \mathrm{~kg}$ de peso corporal, continuando con 2.6 Mcal EM/kg MS hasta el final de la prueba.

AGP= ácidos grasos poliinsaturados; AGM= ácidos grasos monoinsaturados; AGS= ácidos grasos saturados . a,b,c Literales diferentes en el mismo renglón indican diferencia estadística $(P<0.05)$.

Fuente: Bustamante et $a l^{(13)}$.

Por otro lado, un estudio en el que se evaluaron características de canal de toretes ${ }^{(9)}$, estableció que el NE 4 fue diferente $(P<0.05)$ al NE $2(53.1 \mathrm{vs} 51.2 \%$, respectivamente), pero similar $(P>0.05)$ al NE $1(52.0 \%)$ y NE $3(51.9 \%)$, para rendimiento en canal. Sin embargo, para área del ojo de la costilla, grasa renal, pélvica y cardiaca, y rendimiento en cortes magros, el NE 4 fue diferente $(P<0.05)$ al NE 1 (12.3 vs 11.2 pulgadas $^{2}, 3.05$ vs $3.95 \%$, y 50.1 vs $49.2 \%$, respectivamente), pero similar $(P>0.05)$ al NE 2 (11.6 pulgadas ${ }^{2}$, $3.37 \%$ y $48.1 \%$, respectivamente) y NE 3 (11.4 pulgadas $^{2}, 3.45 \%$ y $48.9 \%$, respectivamente) (Cuadro 4). El NE 3 produjo más grasa de cobertura $(P<0.05)$ que el NE 4 
(0.31 vs 0.22 pulgadas), pero estos fueron similares $(P>0.05)$ a los NE 1 y 2 . En contraste, el NE 2 produjo mayor grado de rendimiento $(P<0.05)$ que el NE 4 (2.4 vs 1.6 unidades), pero estos fueron similares $(P>0.05)$ a los NE 1 y 3 (Cuadro 4$)$. El NE 4 produjo mayor porcentaje de cortes primarios y porcentaje de cortes totales $(P<0.05)$ que el NE $2(53.1 v s 51.2 \%$, y 81.2 vs $77.4 \%$, respectivamente), pero estos fueron similares $(P>0.05)$ a los NE 1 y 3 (Cuadro 4). Por lo tanto, en el estudio se concluyó que toretes $C$ alimentados en corral producen canales con notables características de rendimiento y calidad en respuesta a dietas altas en energía, las cuales se utilizan comúnmente en la finalización comercial de bovinos productores de carne.

Cuadro 4: Características de canal de toretes Criollo Coreño alimentados con distintos niveles de energía

\begin{tabular}{lcccc}
\hline \multicolumn{1}{c}{ Característica } & NE 1 & NE 2 & NE 3 & NE 4 \\
\hline Rendimiento de canal, \% & $52.0^{\mathrm{ab}}$ & $51.2^{\mathrm{b}}$ & $51.9^{\mathrm{ab}}$ & $53.1^{\mathrm{a}}$ \\
Área del ojo de la costilla, pulgadas $^{2}$ & $11.2^{\mathrm{b}}$ & $11.6^{\mathrm{ab}}$ & $11.4^{\mathrm{ab}}$ & $12.3^{\mathrm{a}}$ \\
Grasa de cobertura, pulgadas & $0.26^{\mathrm{ab}}$ & $0.25^{\mathrm{ab}}$ & $0.31^{\mathrm{a}}$ & $0.22^{\mathrm{b}}$ \\
Grasa renal, pélvica y cardiaca, \% & $3.95^{\mathrm{b}}$ & $3.37^{\mathrm{ab}}$ & $3.45^{\mathrm{ab}}$ & $3.05^{\mathrm{a}}$ \\
Grado de rendimiento, unidades & $2.0^{\mathrm{ab}}$ & $2.4^{\mathrm{a}}$ & $2.1^{\mathrm{ab}}$ & $1.6^{\mathrm{b}}$ \\
Cortes primarios, \% & $52.0^{\mathrm{ab}}$ & $51.2^{\mathrm{a}}$ & $51.9^{\mathrm{ab}}$ & $53.1^{\mathrm{b}}$ \\
Cortes totales, \% & $79.4^{\mathrm{ab}}$ & $77.4^{\mathrm{b}}$ & $78.7^{\mathrm{ab}}$ & $81.2^{\mathrm{a}}$ \\
Cortes magros, \% & $49.2^{\mathrm{b}}$ & $48.1^{\mathrm{ab}}$ & $48.9^{\mathrm{ab}}$ & $50.1^{\mathrm{a}}$ \\
\hline
\end{tabular}

$\mathrm{NE} 1=2.2 \mathrm{Mcal}$ EM/kg MS de inicio a fin; NE $2=2.4 \mathrm{Mcal}$ EM/kg MS de inicio a fin; NE $3=2.2 \mathrm{Mcal}$ $\mathrm{EM} / \mathrm{kg}$ MS del inicio hasta los $300 \mathrm{~kg}$ de peso corporal, continuando con $2.4 \mathrm{Mcal}$ EM/kg MS hasta el final de la prueba; NE 4= 2.4 Mcal EM/kg MS del inicio hasta los $300 \mathrm{~kg}$ de peso corporal, continuando con 2.6

Mcal EM/kg MS hasta el final de la prueba.

a,b,c Literales diferentes en el mismo renglón indican diferencia $(P<0.05)$.

Fuente: Bustamante $e t a l^{(9)}$.

\section{Caracterización molecular}

Un estudio de la diversidad autosómica entre siete grupos genéticos (criollos de Chihuahua, Durango, Nayarit (Criollo Coreño) y Guerrero; ganado de Lidia; Criollo Lechero Centroamericano; y Guzerat), en el que se utilizaron 9 microsatélites mostró que ha ocurrido una diferenciación genética considerable entre las poblaciones de bovinos criollos de Nayarit, Chihuahua, Durango y Guerrero; además, se encontró que no hay evidencia de influencia de Bos indicus en los bovinos criollos de Nayarit, Chihuahua y Durango, pero si en los de Guerrero $^{(15)}$.

Usando marcadores SNP en plataforma de alta densidad en un estudio de la diversidad genética del ganado C de tres localidades de Nayarit: El Nayar (N), La Yesca (Y) y Santiago Ixcuintla (S), se encontró que estas poblaciones mantienen moderados niveles de heterocigosidad promedio observada (de 0.29 a 0.34 ) y que la coancestría molecular estimada indica que la población de $\mathrm{N}$ es diferente de las otras dos, que pueden considerarse como 
parte de una misma población ${ }^{(16)}$. Por otro lado, los análisis de la variación en el número de copias (CNV) en estas poblaciones resultaron en la identificación de 2,170 CNV en 40 animales, localizadas en 733 regiones, con una cobertura de $32.1 \mathrm{Mb}$ del genoma autosómico. El análisis funcional llevó a la asociación de estas CNV con 131 genes involucrados principalmente en inflamación y respuesta inmune y 923 QTLs superpuestos, clasificados en 6 categorías diferentes de QTL-term: características reproductivas, productivas, de conformación, producción de leche, de la canal y de la carne, y de salud. Los análisis de grupo y componentes principales mostraron que los animales se agruparon de acuerdo con la localidad de origen, aunque mostraron un origen genético común por compartir 75 de las 302 CNV identificadas en más de dos animales. Asimismo, se encontró que los criollos Y compartieron otros 34 y $30 \mathrm{CNV}$ con los $\mathrm{N}$ y $\mathrm{S}$, respectivamente; mientras que $\mathrm{N}$ y $\mathrm{S}$ compartieron $56 \mathrm{CNV}$, lo que sugiere que los criollos $\mathrm{N}$ y S son más cercanos entre ellos, lo que puede deberse a que la población $\mathrm{S}$ se originó en parte de animales traídos de $\mathrm{N}$ hace 25 años. Las CNV propias de cada población fueron 36 para N, 37 para Y, y 34 para S, lo que sugiere la existencia de diversidad genética entre las poblaciones ${ }^{(17)}$.

\section{Retos y perspectivas de investigación}

La conservación de la diversidad de los recursos genéticos animales es importante para la sostenibilidad de los sistemas de producción pecuarios. La variación genética en las poblaciones de ganado en el mundo está disminuyendo, tanto dentro como entre razas. Sin embargo, la variación genética es necesaria para que se mejore genéticamente la productividad y la adaptación a condiciones ambientales cambiantes, como el clima y condiciones de producción o de mercado.

En el caso del ganado Criollo Coreño, la investigación generada en el INIFAP sugiere que este ganado puede jugar un papel destacado como raza materna en el contexto de la producción comercial de carne de bovino. En el corto plazo es importante continuar, de manera sistemática, el registro de la información productiva de interés económico en el hato experimental del S.E. "El Verdineño", así como identificar los genotipos más productivos utilizando arreglos de alta densidad. Esto permitirá que a mediano plazo se puedan 1) identificar regiones genómicas sujetas a selección natural y evidencias de selección artificial con enfoques cuantitativos, y 2) estimar relaciones genómicas entre individuos y niveles de consanguinidad utilizando corridas de homocigosis ${ }^{(18)}$. No se tiene un conocimiento real de la situación de riesgo de este ganado, por lo que es importante, a mediano plazo, hacer una evaluación del tamaño efectivo y estructura de la población y repetirla cada cinco años para tener un monitoreo efectivo que permita conocer la tendencia en el tamaño de la población ${ }^{(19)}$. En el largo plazo, se deben realizar estudios para identificar genes asociados a características de interés presente y futuro. 
En cuanto a otras poblaciones mexicanas de ganado bovino Criollo, en el corto plazo se deben hacer estudios de diversidad genética dentro y entre grupos, utilizando información genética molecular. En el mediano plazo se deben integrar hatos controlados que permitan el registro sistemático de las características productivas y adaptativas de los distintos fenotipos, y en el largo plazo realizar estudios para la identificación de polimorfismos únicos que distingan a estas poblaciones. Debido al tamaño pequeño de las poblaciones de bovino criollo mexicano, a largo plazo se podrían esperar pequeñas ganancias, en término económicos, con la utilización de la información genómica ${ }^{(20)}$; sin embargo, las poblaciones de bovino criollo mexicano se pueden beneficiar de los desarrollos tecnológicos que se obtienen en otras razas, cuyas poblaciones son mayores, como son los métodos para la optimización de la respuesta genética y para el mantenimiento de la diversidad, los cuales pueden ser más fácilmente aplicados en poblaciones pequeñas ${ }^{(21)}$.

\section{Conclusiones}

La información generada por el INIFAP en los últimos 20 años indicó la influencia de $\mathrm{h}_{\mathrm{i}}$ en la reproducción y producción de leche, así como la influencia de $\mathrm{h}_{\mathrm{m}}$ en el crecimiento durante la etapa nacimiento-destete de las crías, lo que se reflejó en mayor productividad al destete por vaca en empadre. Los efectos genéticos directos y maternos no influyeron sobre las características analizadas. Por otro lado, se determinó que toretes $\mathrm{C}$ alimentados con dietas altas en energía en confinamiento producen carne con perfiles favorables de ácidos grasos y canales de buena calidad, y que es factible utilizar dosis reducidas de FSH para la superovulación en vaquillas $\mathrm{C}$, sin afectar la respuesta a la producción de embriones. Las vacas GC son mejor opción que las vacas CG para la producción de becerros para engorda, con respecto a la productividad de las vacas $\mathrm{G}$ o $\mathrm{C}$. Las poblaciones $\mathrm{C}$ mantienen niveles importantes de diversidad genética. Se recomienda, para todas las poblaciones mexicanas de ganado bovino Criollo, hacer estudios de diversidad genética utilizando información genética molecular, e integrar hatos controlados que permitan el registro sistemático de fenotipos con su productividad y adaptabilidad, para poder identificar genes exclusivos en esta población.

\section{Literatura citada:}

1. SAGARPA. Informe sobre la situación de los Recursos Genéticos Pecuarios (RGP) de México. Coordinación General de Ganadería. 2002. http://www.sagarpa.gob.mx/ganaderia/Publicaciones/Paginas/InfoRGPecuariosM.aspx

2. FAO. The management of global animal genetic resources. Proc Expert Consultation, Anim Prod Health. Hodges J, editor. FAO, Rome. Paper No. 104, 1992:1-263.

3. Martínez VG. El ganado bovino Criollo en Nayarit: Ubicación y población estimada. Sitio Experimental "El Verdineño". CIRPAC- INIFAP. Folleto Técnico Número 1. 2005. Nayarit, México. 
4. Dickerson GE. Inbreeding and Heterosis in Animals. Proc Anim Breed Symp, Honor of Jay Lush. Am Soc Anim Sci \& Am Dairy Sci Assoc. Illinois. 1973:54-77.

5. Martínez VG, Montaño BM, Palacios FJA. Efectos genéticos directos, maternos y heterosis individual para tasas de estro, gestación, parición y destete de vacas Criollo, Guzerat y sus cruzas F1. Téc Pecu Méx 2006;44(2):143-154.

6. Martínez VG, Montaño BM, Palacios FJA. Productividad hasta el destete de vacas Criollo, Guzerat y sus cruzas recíprocas F1. Téc Pecu Méx 2008;46(1):1-12.

7. Martínez VG, Montaño BM. El bovino Criollo del Occidente de México. Perezgrovas GRA y De la Torre SF editores. Los Bovinos Criollos de México: Historia, Caracterización y Perspectivas. Primera edición. Tuxtla Gutiérrez, Chiapas, México: Universidad Autónoma de Chiapas; 2015: 147-174. https://www.textosdeinvestigacion.unach.mx/ebooksbd/20151023_121503/\#p=174

8. Martínez VG, Bustamante GJJ, Palacios FJA, Montaño BM. Efectos raciales y heterosis materna Criollo-Guzerat para crecimiento posdestete y características de la canal Criollo Coreño. Téc Pecu Méx 2006;44(1):107-118.

9. Bustamante GJJ, Martínez VG, Basurto GR, Palacios FJA, Moreno FLA, Montaño BM. Rendimiento y calidad de la canal de toretes criollos finalizados en corral [resumen]. V Congreso Internacional de Manejo de Pastizales. I Congreso en Ciencias Veterinarias y Zootécnicas “Amado Nervo". Reunión Red Latina en Ciencia Animal. Nuevo Vallarta, Nayarit. 2014:1-5.

10. Villaseñor GF, de la Torre SJF, Martínez VG, Álvarez GH, Pérez RS, Palacios FJA, Polanco SR, Montaño BM. Caracterización de la respuesta ovárica a la superovulación en bovino Criollo Coreño utilizando dosis reducidas de FSH. Rev Mex Cienc Pecu 2017;8(3):225-232.

11. Martínez VG, Borrayo ZA, Montaño BM, Bustamante GJJ, Palacios FJA, Vega MVE, Ríos UA. Producción de leche de vacas Criollo, Guzerat y sus cruzas recíprocas F1 y su relación con el peso al destete de las crías. Rev Mex Cienc Pecu 2012;3(4):501-514.

12. Martínez VG, Palacios FJA, Bustamante GJJ, Ríos UA, Vega MVE, Montaño BM. Composición de leche de vacas Criollo, Guzerat y sus cruzas F1 y su relación con el peso al destete de las crías. Rev Mex Cienc Pecu 2010;1(4):311-324.

13 Bustamante GJJ, Martínez VG, Palacios FJA, Basurto GR. Perfil de ácidos grasos en carne de toretes Criollo alimentados con distintos niveles de energía en la dieta [resumen]. V Congreso Internacional de Manejo de Pastizales. I Congreso en Ciencias Veterinarias y Zootécnicas “Amado Nervo". I Reunión Red Latina en Ciencia Animal. Nuevo Vallarta, Nay. 2014;1-6. 
14. Simopoulos AP. Evolutionary aspects of diet, the omega-6/omega-3 ratio and genetic variation: nutritional implications for chronic diseases. Biomed Pharmacother 2006;60:502-507.

15. Ulloa-Arvizu R, Gayosso-Vázquez A, Ramos-Kuri M, Estrada FJ, Montaño M, Alonso RA. Genetic analysis of Mexican Criollo cattle populations. J Anim Breed Genet 2008;125(5):351-359.

16. Martínez RCP, Martínez VG, Román PSI, Cortes CM, Guzmán RLF, De la Torre SJF, et al. Estimación de la diversidad genética mediante marcadores SNP en bovino Criollo Coreño (Bos taurus) [resumen]. Reunión Nacional de Investigación Pecuaria. Toluca, Edo. De México. 2015:189-191.

17. Cozzi MC, Martinez-Ruiz CP, Roman-Ponce SI, Vega-Murillo VE, Ríos-Utrera A, Montaño-Bermúdez $\mathrm{M}$, et al. Copy number variants reveal genomic diversity in a Mexican Creole cattle population. Livest Sci 2019;229:194-202. doi.org/10.1016/j.livsci.2019.09.030.

18. Purifield DC, Berry DP, McParland S, Bradley DG. Runs of homozygosity and population history in cattle. BMC Genet 2012;13:70. doi:10.1186/1471-2156-13-70.

19. Boettcher PJ, Tixier-Boichard M, Toro MA, Simianer H, Eding H, Gandini G, et al, GLOBALDIV Consortium. Objectives, criteria and methods for using molecular genetic data in priority setting for conservation of animal genetic resources. Anim Genet 2010; 41 (Suppl. 1):64-77.

20. Pryce JE, Daetwyler HD. Designing dairy cattle breeding schemes under genomic selection: a review of international research. Anim Prod Sci 2012;52(3):107-114.

21. Gandini G, Del Corvo M, Biscarini F, Stella A. Genetic improvement of small ruminant local breeds with nucleus and inbreeding control: a simulation study. Small Ruminant Res 2014; 120:196-203. doi:10.1016/j.smallrumres. 2014.06.004. 